\title{
Therapeutic and Prophylactic Potential of Small Interfering RNAs against Severe Acute Respiratory Syndrome Progress to Date
}

\author{
Zhijie Chang, ${ }^{1}$ Lorne A. Babiuk ${ }^{2}$ and Jim $\mathrm{Hu}^{3}$
}

1 School of Medicine, Department of Biological Sciences and Biotechnology, Tsinghua University, Beijing, China

2 Vaccine \& Infectious Disease Organization (VIDO), University of Saskatchewan, Saskatoon, Saskatchewan, Canada

3 Lung Biology Research Program, Hospital for Sick Children Research Institute, and University of Toronto, Toronto, Ontario, Canada

\begin{abstract}
Severe acute respiratory syndrome (SARS), caused by the novel coronavirus SARS-CoV, produced a scare when it appeared in 2003 in China and later quickly spread to other countries around the world. Although it has since disappeared, its threat to human health remains. Therefore, studies on the prevention and treatment of SARS are important for dealing with epidemics of this and other infectious diseases. The most promising newly developed technology for intervention in SARS may be RNA interference, an endogenous cellular process for the inhibition of gene expression mediated by sequence-specific double-stranded RNAs. Numerous studies have reported the therapeutic potential of RNA interference for the treatment of various human diseases ranging from cancers to infectious diseases such as HIV and hepatitis. To date, most studies on inhibition of SARS-CoV replication using small interfering RNAs (siRNAs) have been conducted in cell lines in vitro. One study using siRNAs to inhibit SARS-CoV infection in Rhesus macaques demonstrated that siRNAs were effective both prophylactically and therapeutically with no adverse effects in the animals. Challenges remaining for the application of siRNA in vivo for SARS prevention and treatment include the specificity of the siRNAs and the efficiency of delivery. However, with improvements in siRNA design and delivery methods, RNA interference has the potential to become another major weapon for combating dangerous infections due to viruses such as SARS-CoV.
\end{abstract}

During the 2003-4 severe acute respiratory syndrome (SARS) epidemic, ${ }^{[1]}$ there was a high mortality rate ${ }^{[2]}$ and there were no rational methods for treatment. Empirical practices for treating the typical influenza-like symptoms were applied, such as using antibiotics, antiviral agents (ribavirin, oseltamivir, and HIV-protease inhibitors), corticosteroids, interferons, normal human immunoglobulin preparations, and Chinese herbs. ${ }^{[3]}$ Molecular diagnostic studies confirmed that a novel coronavirus, named SARS-CoV (the SARS-associated coronavirus), was responsible for the disease. ${ }^{[4,5]}$ This led to the search for rational regimens to treat the infection, including the generation of vaccines ${ }^{[6-8]}$ and the design of inhibitors of SARS-CoV replication. ${ }^{[9]}$ The most promising newly developed technology for intervention in SARS may be RNA interference (RNAi). ${ }^{[10]}$

SARS-CoV, as a member of the coronavirus family, is a singlestranded plus-sense RNA virus with a membrane envelope. ${ }^{[11]}$ The SARS-CoV genome is approximately $30 \mathrm{~kb}$ in length and encodes a consensus leader sequence, the polyproteins $1 \mathrm{a}$ and $1 \mathrm{~b}$, and at least four major structural proteins including the $\mathrm{N}$ (nucleocapsid), $\mathrm{S}$ (spike), M (membrane), and $\mathrm{E}$ (small envelope) proteins. The four structural proteins constitute the virus particle. The $\mathrm{N}$ protein is a virion RNA-associated protein and forms a long and flexible helical nucleocapsid; this protein is also important for viral RNA synthesis. The $\mathrm{S}$ protein is a glycoprotein on the viral surface and is responsible for viral attachment and entry into host cells. The $\mathbf{M}$ 
protein is necessary for virus budding and the E protein for virus assembly.

SARS-CoV replication requires first a partial duplication of its genome and the translation of some genome-encoded proteins. This leads to the first synthesis of a consensus leader sequence, a sub-genome, and the polyproteins $1 \mathrm{a}$ and $1 \mathrm{~b}$. The leader sequence is capped into the transcripts from the SARS-CoV sub-genome and plays a role in translation of the structural proteins. ${ }^{[4]}$ Polyproteins $1 \mathrm{a}$ and $1 \mathrm{~b}$ are directly synthesized from the viral genome without the leader sequence. These proteins include papain-like cysteine protease (PLpro), 3C-like cysteine protease (3CLpro), and RNA-dependent RNA polymerase (RDRP), which are required for viral replication and transcription. ${ }^{[4]}$ Each step in the key processes during viral replication can be a potential target for the interruption of SARS-CoV replication. Since SARS-CoV replication also requires certain host proteins, genes from host cells involved in viral replication can also be selected as targets.

\section{Small Interfering RNA (siRNA) and its Application}

RNA interference is an endogenous cellular process for the inhibition of gene expression mediated by sequence-specific double-stranded RNAs (dsRNAs). This phenomenon was first observed in petunia flowers, and later reported in Caenorhabditis elegans. ${ }^{[12]}$ These dsRNAs, $21-23$ bp in length, are produced by cleavage of a long RNA duplex by Dicer, a ribonuclease III-type enzyme. When produced, the small interfering RNA (siRNA) duplexes can activate a process of degradation of mRNA transcripts containing target sequences. This process is driven by the siRNA-enzyme complex or RNA-induced silencing complex (RISC) and results in inhibition of gene expression. Interestingly, when dsRNAs are artificially generated either by direct chemical synthesis or by folding single RNA into short dsRNAs with a hairpin loop, they can target the specific mRNA sequences and induce degradation of the mRNA. Therefore, RNA interference can be a tool for down-regulation of gene expression in cultured cells as well as in living organisms. ${ }^{[13,14]}$

The two most important issues that must be considered before the application of siRNAs to down-regulation of gene expression becomes a reality are: (i) generation of effective dsRNAs; and (ii) delivery of the dsRNAs into targeted cells or organs. To address the first issue, researchers have established two strategies. The first one is to chemically synthesize siRNA. ${ }^{[15]}$ In this method, two single-stranded, complementary, short RNA oligonucleotides are synthesized. These two short oligonucleotides are then annealed into a dsRNA in a test tube. Usually short oligonucleotides (21-23 nucleotides) are designed with a two-nucleotide overhang to facilitate the activation of the RISC during the process of mRNA cleavage. In addition, a $3^{\prime}$-hydroxyl group and a $5^{\prime}$-phosphate group are generated to enhance the function of the siRNA duplex. ${ }^{[16]}$ While it is simple and quick in its application, this chemical synthesis strategy has shown limitations in both stability and production cost of the siRNA.

To overcome the limitations of chemical synthesis of siRNA, a plasmid-based strategy was developed. In this strategy, a dsRNA of $21 \mathrm{bp}$ with a hairpin loop of nine nucleotides is designed to be expressed from a plasmid under the control of a promoter. ${ }^{[17]}$ In cells, such RNA molecules are subjected to cleavage by Dicer to generate siRNAs. Since DNA plasmids can be easily and economically produced in Escherichia coli, this strategy has been widely adopted for the characterization of gene functions in vitro and for therapeutic studies. ${ }^{[14]}$

To address the issue related to delivery of siRNAs into cells or living organisms, researchers have used several approaches, including: (i) direct introduction of the chemically synthesized siRNAs; (ii) employment of liposome-conjugated siRNAs or plasmids; and (iii) application of viral vectors to express dsRNAs with a hairpin loop. Each of the methods has both advantages and disadvantages. Recently, numerous papers have reported siRNA technology as a potent tool to knock-down gene expression or its therapeutic potential for treatment of various human diseases ranging from cancers ${ }^{[18]}$ to infectious diseases (e.g. HIV, hepatitis). ${ }^{[14]}$ In this review, we focus on the application of siRNA technology to SARS. Readers are also referred to another recent review on this subject. ${ }^{[19]}$

\section{Progress in the Utilization of siRNAs Against the Severe Acute Respiratory Syndrome-Associated Coronavirus}

As the genomic sequence of SARS-CoV was determined and its mechanism of replication became apparent (similar to others in this coronavirus family), siRNAs targeting different genes of SARS-CoV were used by various groups to inhibit virus gene expression and thereafter to inhibit replication of the virus (see table I). The initial report came from two groups using the chemical synthesis strategy. ${ }^{[20,21]}$ The first group showed that two of six chemically synthesized siRNAs, based on the SARS-CoV genome sequence, resulted in the inhibition of viral cytopathic effect (CPE) in Vero-E6 cells infected with strain Y3-SARS-CoV. ${ }^{[21]}$ The cursory evaluation of the CPE of the transfected cells provided evidence that siRNA might be effective in inhibiting SARS-CoV replication. The second group demonstrated that three of six siRNAs designed to target the replicase A of SARS-CoV were moderately effective, whereas the other three were less effective. ${ }^{[20]}$ 
Table I. A summary of the small interfering RNAs designed to target sequences of the severe acute respiratory syndrome-associated coronavirus (SARSCoV)

\begin{tabular}{|c|c|c|c|c|}
\hline Target & Sequence & Method & Validation & References \\
\hline Replicase $1 \mathrm{~A}$ & $5^{\prime}$-gcacuugucuaccuugaug-3' & Chemical synthesis & FRhK-4, GZ50 strain & 20 \\
\hline Genome $^{a}$ & $\begin{array}{l}\text { 5'-ccaucaagccugugucguaTT-3' } \\
5^{\prime} \text {-caucaaauugcgcuaagag TT-3' }\end{array}$ & Chemical synthesis & Strain Y3-SARS-CoV & 21 \\
\hline $\begin{array}{l}\text { Spike1 } \\
\text { Spike2 }\end{array}$ & $\begin{array}{l}5^{\prime} \text {-gagacatatctaatgtgcc-3' } \\
5^{\prime} \text {-gggctaccaccttatgtcc-3' }\end{array}$ & pBS/U6-Vector & $\begin{array}{l}\text { Vero E6 cells, } \\
\text { strain BJ01 }\end{array}$ & 22 \\
\hline RNA polymerase & $\begin{array}{l}5^{\prime} \text {-cttacatagctcgcgtctc-3' } \\
5^{\prime} \text {-gaatattaggcgcaggctg-3' }\end{array}$ & pSUPER.retro & $\begin{array}{l}\text { Vero E6 cells, strain } \\
\text { HKU-39849 }\end{array}$ & 23 \\
\hline Leader & $5^{\prime}$-ccaaccaacctcgatctc- $3^{\prime}$ & pBS/U6-Vector & Vero E6, BJ01 & 24 \\
\hline Spike & $\begin{array}{l}5^{\prime} \text {-aatttgtcaatctcaaagctc-3' } \\
5^{\prime} \text {-aaagaaacagcaaagaaaggg-3' }\end{array}$ & Chemical synthesis & $\begin{array}{l}\text { HEK293T, } \\
\text { EGFP-S }\end{array}$ & 25 \\
\hline RNA polymerase & $5^{\prime}$-atcttaggattgcctacgc- $3^{\prime}$ & pSilencer1.0-U5 & $\begin{array}{l}\text { Vero E6, strain SARS CoV- } \\
\text { p9 }\end{array}$ & 26 \\
\hline $\begin{array}{l}\text { Spike } \\
\text { Spike } \\
\text { 3'-UTR }\end{array}$ & $\begin{array}{l}5^{\prime} \text {-gggctatcaacctatagatdTdT-3' } \\
5^{\prime} \text {-caaggcgattagtcaaattdTdT-3' } \\
5^{\prime} \text {-cgtaactaaacagcacaagdTdT-3' }\end{array}$ & Chemical synthesis & $\begin{array}{l}\text { Vero E6, } \\
\text { Hong Kong strain }\end{array}$ & 27 \\
\hline Leader & $\begin{array}{l}5^{\prime} \text {-gatggagagccttgttctt-3' } \\
5^{\prime} \text {-cagccctatgtgttcatta-3' } \\
5^{\prime} \text {-ctcactcgtgagctcaatg-3' }\end{array}$ & pSilencer3.1-H1 & Vero E6, strain BJ01 & 28 \\
\hline Nucleocapsid & $5^{\prime}$-ggcatcgtatgggttgcaact-3' & Cassette, pMD-18T & Mouse muscle, N-EGFP & 29 \\
\hline $\begin{array}{l}\text { Envelope } \\
\text { Nucleocapsid } \\
\text { Membrane }\end{array}$ & $\begin{array}{l}\text { 5'-ugaaggaguuccugaucuucuTT-3' } \\
5^{\prime} \text {-agcuuaaacaacuccuggaacTT-3' } \\
5^{\prime} \text {-gauaauggacccaaucaaacTT-3' }\end{array}$ & Chemical synthesis & $\begin{array}{l}\text { Vero E6, } \\
\text { E-EGFP, N-EGFP, M-EGFP }\end{array}$ & 30 \\
\hline Genome & $\begin{array}{l}\text { 5'-gcuccuaauuacacucaacdTdT-3' } \\
5^{\prime} \text {-ggaugaggaaggcaauuuadTdT-3' }\end{array}$ & $\begin{array}{l}\text { Chemical synthesis, C5W } \\
\text { solution }\end{array}$ & $\begin{array}{l}\text { Rhesus macaques, strain } \\
\text { PUMC01 }\end{array}$ & 31,32 \\
\hline $\begin{array}{l}\text { Spike } \\
\text { Envelope } \\
\text { Nucleocapsid } \\
\text { Membrane } \\
\text { Membrane }\end{array}$ & $\begin{array}{l}\text { 5'-cacugauuccguucgagauc-3' } \\
5^{\prime} \text {-cguuucggaagaaacagguac-3' } \\
5^{\prime} \text {-caagccucuucucgcuccuc-3' } \\
5^{\prime} \text {-ugcuugcugcugucuacag-3' } \\
5^{\prime} \text {-guggcuuagcuacuucguug-3' }\end{array}$ & $\begin{array}{l}\text { Chemical synthesis, } \\
\text { combination use }\end{array}$ & FRhK-4, Strain GZ50 & 33 \\
\hline $\begin{array}{l}\text { Genome } \\
\text { RdRP }\end{array}$ & $\begin{array}{l}\text { 5'-ggaugaggaaggcaauuuaTT-3' } \\
5^{\prime} \text {-cugguacgauuucggugauTT-3' } \\
5^{\prime} \text {-acugucaaacccgguaauuTT-3' }\end{array}$ & $\begin{array}{l}\text { Chemical synthesis, LNA } \\
\text { modified }\end{array}$ & $\begin{array}{l}\text { Luc reporter, HEK293, Strain } \\
\text { Frankfurt } 1\end{array}$ & 34 \\
\hline $\begin{array}{l}\text { Envelope } \\
\text { RdRP }\end{array}$ & $\begin{array}{l}5^{\prime} \text {-aaggagttcctgatcttctggt-3' } \\
5^{\prime} \text {-aaggacatgacctaccgtagac-3' }\end{array}$ & $\begin{array}{l}\text { Vector (modifed U6 } \\
\text { promoter) }\end{array}$ & $\begin{array}{l}\text { envE-pcDNA3.1/ } \\
\text { RDRPpcDNA3.1, NIH3T3 }\end{array}$ & 35 \\
\hline
\end{tabular}

a Sequence information was acquired from the authors.

LNA = locked nucleic acid.

Around at the same time, Zhang et al. ${ }^{[22]}$ used a vector-based strategy and designed two different siRNAs targeting the sequences encoding the $\mathrm{S}$ protein of SARS-CoV. These sequences were chosen because they were identical in different SARS-CoV strains. The authors inserted the siRNA sequences into the pBS/U6 vector with a U6 promoter, which expresses siRNAs in cells. This group of researchers was the first to demonstrate that the siRNAs inhibited $\mathrm{S}$ gene expression and virus replication in Vero E6 cells infected with SARS-CoV strain BJ01. This study provided strong evidence that siRNAs targeting the $\mathrm{S}$ gene could be employed as potential tools to inhibit SARS-CoV replication. Even though the $S$ protein has a high mutation rate, ${ }^{[36]}$ targeting its mRNA has also been reported by other groups to be effective in the inhibition of SARS-CoV replication. ${ }^{[25,27]}$

Meanwhile, Wang et al. ${ }^{[23]}$ adopted a similar strategy by targeting the viral RNA polymerase gene of SARS-CoV. This group used a commercially available vector pSUPER.retro and designed six siRNAs. These researchers observed that in Vero E6 cells infected with the SARS-CoV strain HKU-39849, two of the six 
siRNAs had a dramatic effect on the inhibition of viral replication. The effectiveness of targeting the RNA polymerase gene was further confirmed by Lu et al. ${ }^{[26]}$ and Meng et al. ${ }^{[35]}$

To avoid the tedious work of constructing plasmid vectors to test effective target sequences, Zheng et al. ${ }^{[31]}$ chemically synthesized 48 siRNA duplexes targeting regions throughout the entire SARS-CoV RNA genome, including the open reading frames for several key proteins. They transfected these siRNA duplexes into fetal Rhesus kidney (FRhK-4) cells prior to, or after, infection with the virus, and evaluated the efficiency of the siRNAs on inhibition of viral replication. They found that four of the siRNA duplexes had a potent inhibitory effect on SARS-CoV infection and replication, evaluated by intracellular viral genome copy number and viral titers in the cell culture medium using quantitative reverse transcription PCR (Q-RT-PCR) and CPE-based titration, respectively. In this study, a prolonged prophylactic effect of siRNA duplexes with up to $90 \%$ inhibition that lasted for at least 72 hours was observed. Combination of active siRNA duplexes, targeting different regions of the viral genome, resulted in up to $80 \%$ inhibition in vitro.

Because they are convenient and rapid to generate, the chemically synthesized siRNA duplexes seem attractive for the identification of targets. Following the study by Zheng et al., ${ }^{[31]}$ Shi et al. ${ }^{[30]}$ synthesized 26 siRNA duplexes and showed that three of these effectively inhibited the expression of a reporter gene carrying a target sequence in Vero E6 cells. To improve the effectiveness of inhibition, this group adopted a strategy utilizing a mutated nucleotide at the last pair of the duplex (with a TT overhang). They found this modification of the duplex increased the inhibitory effects on SARS-CoV gene expression. This observation confirmed that a mismatch in the $5^{\prime}$ end of the antisense siRNA can enhance its effectiveness. ${ }^{[37,38]}$

Recently, more siRNAs to inhibit SARS-CoV gene expression and replication have been reported. Wu et al. ${ }^{[27]}$ described three chemically synthesized siRNA duplexes targeting the $\mathrm{S}$ gene and the 3'-UTR sequences (si-SARS-S2, siSARS-S3, and siSARS-3'UTR2). Zhao et al. ${ }^{[29]}$ designed several siRNAs targeting the sequence encoding the $\mathrm{N}$ protein and, more recently, Meng et al. ${ }^{[35]}$ identified siRNAs targeting the E and RDRP genes.

Interestingly, two groups have reported the effects of targeting the leader sequence of SARS-CoV. ${ }^{[24,28]}$ Li et al. ${ }^{[24]}$ first identified the same leader sequence in the mRNA encoding different structural proteins. Based on this finding, they reasoned that siRNAs targeting the leader sequence should effectively inhibit viral replication in cell culture systems, since they would target a number of proteins simultaneously. This research group provided evidence that siRNA targeting the leader sequence, via a U6 promoter-based vector, had a stronger effect on the inhibition of SARS-CoV replication than siRNAs targeting the $\mathrm{S}$ gene. Later, $\mathrm{Ni}$ et al. ${ }^{[28]}$ selected three of 32 potential siRNA sequences targeting the leader sequence. They demonstrated that three siRNAs delivered in the pSilencer3.0-H1 vector dramatically inhibited SARS-CoV replication as measured by either plaque formation or levels of mRNAs and proteins ( $\mathrm{S}$ and $\mathrm{N}$ ). While another group did not find any significant inhibitory effect of the chemically synthesized siRNA duplex targeting the leader sequence, ${ }^{[27]} \mathrm{Li}$ et al. ${ }^{[24]}$ and $\mathrm{Ni}$ et al. ${ }^{[28]}$ have separately demonstrated the effectiveness of vectordriven siRNAs targeting the leader sequence. Whether there is a difference between the vector-driven and the chemically synthesized siRNAs remains unclear.

The rational design for effective siRNA inhibition of SARS$\mathrm{CoV}$ replication remains difficult, as most authors have selected target sequences mainly by empirical testing. The selected target sequences usually have some specific features, as previous reports have suggested. ${ }^{[14,39]}$ For the ease of selection of siRNA target sequences, Meng et al. ${ }^{[35]}$ adopted a cell-based assay to screen for siRNAs to block SARS-CoV gene expression. This assay involved two genes encoding RDRP and the envelope E protein, respectively. These authors verified the published sequences and validated two siRNA sites for the RDRP gene and two sites for the envelope E gene. This assay provided a specific strategy to select effective siRNA sequences.

Considering that the virus may possibly escape from siRNA targeting through viral mutations, ${ }^{[40,41]}$ He et al. ${ }^{[33]}$ used a combination of siRNAs targeting different sequences. These authors reduced the dosage of the single siRNA duplexes to a minimal effective level and then tested the synergistic effect of the combination of the different duplexes. They found that the combination of siRNAs targeting two different sites had a significant synergistic effect on the inhibition of SARS-CoV replication in infected FRhk-4 cells. However, it is still unclear as to whether this synergistic role could overcome the problem of viral escape through mutation. In this study, they did not observe any synergistic effect of the combination of the siRNA duplexes at high dose, although this may be explained by a saturated dosage effect. ${ }^{[20,31]}$

Currently, researchers have used both chemical synthesis and vector-based approaches to generate siRNAs for SARS-CoV. While the chemical synthesis approach is more rapid and easy to test, the RNA stability is problematic. To improve the biostability, Elmen et al. ${ }^{[34]}$ used a locked nucleic acid (LNA) approach for the design of siRNAS. As reported by Braasch et al., ${ }^{[15]}$ LNA could increase the thermal stability of siRNA duplexes without any deleterious effect on their function. The study by Elmen et al. ${ }^{[34]}$ indicated that incorporation of LNA significantly enhanced siRNA stability and improved the efficiency of the unmodified siRNA on SARS-CoV inhibition. This study demonstrated that the modifica- 
tion of the siRNA duplex could substantially improve its activity. Therefore, this approach may enhance the development of siRNAs as a therapeutic intervention for SARS-CoV.

To date, most studies on the inhibition of SARS-CoV replication using siRNAs have been conducted using cell lines in vitro. However, in the only animal study to date, Li et al. ${ }^{[32]}$ have reported their attempts at using siRNAs to inhibit SARS-CoV infection in Rhesus macaques. After initial screening, two chemically synthesized siRNAs were selected from 48 siRNAs based on the previous work of Zheng et al. ${ }^{[31]}$ The researchers treated the animals with the two siRNA duplexes in a commonly used glucose solution D5W prior to, and after, infection with SARS-CoV and found that all of the monkeys in the treated groups showed less severe symptoms than those in the control groups. They reported that during a 20-day period of observation after the administration of the siRNA duplexes, the treated monkeys showed an average body temperature of $<38.9^{\circ} \mathrm{C}$, while the control animals had an average body temperature of $>39.1^{\circ} \mathrm{C}$. The temperature changes were correlated with the changes in lung histopathology. The intriguing observation was that three of the four animals in each of the three siRNA-treated groups were free of SARS-CoV in the oropharyngeal swab specimens, based on measurements of SARS$\mathrm{CoV}$ RNA copy numbers. This indicated that the SARS-CoV particles released from the animals' lungs were dramatically reduced by the injection of the siRNA duplexes. Whether siRNA inhibited SARS-CoV replication or blocked the spread of the virus within the lungs remains to be determined. However, this study clearly demonstrated that siRNAs were effective both prophylactically and therapeutically with no adverse effects in the animal study. ${ }^{[10]}$ The reduction of virus in the lungs may dramatically reduce spread to contacts, thereby being an effective approach to reducing spread of the virus between patients and caregivers.

\section{Challenges to In Vivo Application of siRNA}

Two challenges remain for the application of siRNA in vivo for SARS prevention and treatment. These involve the specificity of the siRNAs and the efficiency of their delivery.

\subsection{Specificity and Possible Adverse Effects}

The target specificity of siRNA is an important feature of this novel technology for therapeutic development. Highly specific siRNAs can be selected through screening to avoid potential offtarget side effects. However, this high specificity can also be a problem since a single nucleotide change will diminish the effectiveness of the designed siRNA. Since mutations of the SARS$\mathrm{CoV}$ genome can occur, ${ }^{[36]}$ which may result in viral escape from siRNA targeting, ${ }^{[40,41]} \mathrm{He}$ et al. ${ }^{[33]}$ used a combination of different
siRNAs targeting different sequences. This had a further beneficial effect since it appears that the combination of the different siRNAs had a synergistic effect on the inhibition of SARS-CoV replication. Based on this data, it appears that the best approach would be to use a combination of siRNAs for maximal benefit; therefore, we suggest that highly effective siRNAs in combination should be used.

One possible adverse effect of in vivo application is that some siRNAs may trigger host immune responses such as the induction of interferon. ${ }^{[42]}$ However, since most siRNAs do not show this adverse effect, it is possible to conduct screening of siRNA sequences in animals before undertaking clinical studies. Another potential problem with the application of this technology in vivo is that when siRNAs are overexpressed, they may interfere with endogenous microRNA processing. ${ }^{[43]}$ This problem can be avoided by lowering the delivery dose or siRNA expression.

\subsection{Delivery of siRNA}

All the studies referred to in this review have demonstrated potent inhibitory effects of siRNAs (both chemically synthesized and vector based) on the replication of SARS-CoV in cultured cells or in animals. However, for clinical applications in both the prophylactic and therapeutic contexts, it is critical to improve the efficiency of siRNA delivery into specific human organs. As in the gene therapy field, ${ }^{[44]}$ siRNA targeting SARS-CoV is hampered by barriers to delivery. In the work done in Rhesus macaques, Li et al. ${ }^{[32]}$ adopted a very common glucose solution D5W to deliver the chemically synthesized siRNAs into the airways of animals. Although the experimental results demonstrated dramatic changes in SARS-CoV replication, it remained unclear how much of the siRNA duplexes had been successfully delivered into the airway epithelial cells of the animals, and how long the in vivo transfected siRNA duplexes were maintained in the cells. Clearly, this is an area where additional work is needed. However, we are confident that with all the recent work in gene targeting and vaccine formulation, this hurdle is not insurmountable.

Approaches for gene delivery to airways have been greatly improved over the past decade, especially those using viral vectors, such as the helper-dependent adenoviral vector (HDAd) which can transduce almost all the airway epithelial cells in mice and to a lesser degree in rabbits. ${ }^{[45-47]}$ Delivery of siRNAs with viral vectors should be more effective for prophylactic use, since expression of siRNAs from viral vectors is expected to last much longer than the duration of chemically synthesized siRNA in transfected cells. Although viral vectors can be used to deliver siRNAs for SARS-CoV, potential adverse effects, such as inflammation, have to be considered. In fact, siRNAs can be used to 
down-regulate the expression of proinflammatory cytokines. For example, Cao et al. ${ }^{[48]}$ have shown that interleukin (IL)-8 expression can be down-regulated by an HDAd expressing an siRNA targeting IL-8 mRNA in airway epithelial cells. The concern of host immune responses to HDAd may be addressed by using antiinflammatory drugs. In addition, adeno-associated viral vectors that do not cause host immune responses have been shown to be highly efficient in expressing siRNA in mouse livers ${ }^{[33]}$ and may be adapted to airway siRNA delivery as well. Therefore, these viral delivery approaches may be useful to translate RNAi technology into clinical applications.

To date, few studies have been performed to compare the efficiency of siRNA delivery in vitro with that in vivo; more animal studies will be helpful in screening for siRNAs with therapeutic potential. For the rational design of siRNAs for treatment of SARS, we can also learn from experience with the application of siRNAs to other diseases. In this regard, Schiffelers and Storm ${ }^{[49]}$ have reported a system named ICS-283, which was developed for systemic delivery of therapeutic siRNA to sites of pathological angiogenesis. In addition, Dykxhoorn and Leiberman ${ }^{[50]}$ recently commented on different approaches for the intravenous delivery of siRNAs in clinical applications. With the improvement of siRNA design and delivery methods, RNA interference will offer another major weapon for combating dangerous infections by viruses, such as SARS-CoV.

\section{Conclusion}

In this review, we have summarized the application of siRNAs to the prevention and treatment of SARS. Although there are still a number of problems that need to be overcome before this technology can be adopted in clinical applications, we believe that the progress in siRNA methodology will help us develop effective drugs to conquer this and other dangerous infectious diseases.

\section{Acknowledgments}

The authors were supported in part by grants from CIHR, CCFF and FFBC to Jim Hu, and NSFC (30530420) and 973 projects (2001CB510006, 2002CB513007) to Zhijie Chang as well as grants from a China-Canada Joint Health Research Initiative to Jim Hu (\#CCI-82411) and Zhijie Change (\#30611120522).

Jim $\mathrm{Hu}$ is in the Lung Biology Research Program, Hospital for Sick Children Research Institute, Toronto, Ontario, Canada (e-mail: jim.hu@utoronto.ca). Zhijie Chang is at the School of Medicine, Tsinghua University, Beijing, China (e-mail: zhijiec@tsinghua.edu.cn). Lorne Babiuk is a Canada Research Chair in Vaccinology and Biotechnology at the Vaccine and Infectious Disease Organization in Saskatoon, Saskatchewan, Canada (email: lorne.babiuk@usask.ca).

The authors have no conflicts of interest that are directly relevant to the content of this review.

\section{References}

1. Parry J. SARS virus identified, but the disease is still spreading. BMJ 2003 Apr 26; 326 (7395): 897

2. Skowronski DM, Astell C, Brunham RC, et al. Severe acute respiratory syndrome (SARS): a year in review. Annu Rev Med 2005; 56: 357-81

3. Cinatl J Jr, Michaelis M, Hoever G, et al. Development of antiviral therapy for severe acute respiratory syndrome. Antiviral Res 2005 Jun; 66 (2-3): 81-97

4. Holmes KV. SARS-associated coronavirus. N Engl J Med 2003 May 15; 348 (20): 1948-51

5. Drosten C, Gunther S, Preiser W, et al. Identification of a novel coronavirus in patients with severe acute respiratory syndrome. N Engl J Med 2003 May 15; 348 (20): 1967-76

6. Jiang S, He Y, Liu S. SARS vaccine development. Emerg Infect Dis $2005 \mathrm{Jul} ; 11$ (7): 1016-20

7. Zhi Y, Wilson JM, Shen H. SARS vaccine: progress and challenge. Cell Mol Immunol 2005 Apr; 2 (2): 101-5

8. Taylor DR. Obstacles and advances in SARS vaccine development. Vaccine 2006 Feb 13; 24 (7): 863-71

9. Kesel AJ. Synthesis of novel test compounds for antiviral chemotherapy of severe acute respiratory syndrome (SARS). Curr Med Chem 2005; 12 (18): 2095-162

10. Chang Z, Hu J. RNAi therapeutics: Can siRNAs conquer SARS? Gene Ther 2006 Jun; 13 (11): 871-2

11. Marra MA, Jones SJ, Astell CR, et al. The genome sequence of the SARSassociated coronavirus. Science 2003 May 30; 300 (5624): 1399-404

12. Fire A, Xu S, Montgomery MK, et al. Potent and specific genetic interference by double-stranded RNA in Caenorhabditis elegans. Nature 1998 Feb 19; 391 (6669): 806-11

13. Sharp PA. RNA interference: 2001. Genes Dev 2001 Mar 1; 15 (5): 485-90

14. Shi Y. Mammalian RNAi for the masses. Trends Genet 2003 Jan; 19 (1): 9-12

15. Braasch DA, Jensen S, Liu Y, et al. RNA interference in mammalian cells by chemically-modified RNA. Biochemistry 2003 Jul 8; 42 (26): 7967-75

16. Dykxhoorn DM, Novina CD, Sharp PA. Killing the messenger: short RNAs that silence gene expression. Nat Rev Mol Cell Biol 2003 Jun; 4 (6): 457-67

17. Sui G, Soohoo C, Affar el B, et al. A DNA vector-based RNAi technology to suppress gene expression in mammalian cells. Proc Natl Acad Sci U S A 2002 Apr 16; 99 (8): 5515-20

18. Pai SI, Lin YY, Macaes B, et al. Prospects of RNA interference therapy for cancer. Gene Ther 2006 Mar; 13 (6): 464-77

19. Wu CJ, Chan YL. Antiviral applications of RNAi for coronavirus. Expert Opin Investig Drugs 2006 Feb; 15 (2): 89-97

20. He ML, Zheng B, Peng Y, et al. Inhibition of SARS-associated coronavirus infection and replication by RNA interference. JAMA 2003 Nov 26; 290 (20): 2665-6

21. Zhang R, Guo Z, Lu J, et al. Inhibiting severe acute respiratory syndromeassociated coronavirus by small interfering RNA. Chin Med J (Engl) 2003 Aug; 116 (8): 1262-4

22. Zhang $\mathrm{Y}, \mathrm{Li} \mathrm{T}, \mathrm{Fu} \mathrm{L}$, et al. Silencing SARS-CoV spike protein expression in cultured cells by RNA interference. FEBS Lett 2004 Feb 27; 560 (1-3): 141-6

23. Wang Z, Ren L, Zhao X, et al. Inhibition of severe acute respiratory syndrome virus replication by small interfering RNAs in mammalian cells. J Virol 2004 Jul; 78 (14): 7523-7

24. Li T, Zhang Y, Fu L, et al. siRNA targeting the leader sequence of SARS-CoV inhibits virus replication. Gene Ther 2005 May; 12 (9): 751-61

25. Qin ZL, Zhao P, Zhang XL, et al. Silencing of SARS-CoV spike gene by small interfering RNA in HEK 293T cells. Biochem Biophys Res Commun 2004 Nov 26; 324 (4): 1186-93

26. Lu A, Zhang H, Zhang X, et al. Attenuation of SARS coronavirus by a short hairpin RNA expression plasmid targeting RNA-dependent RNA polymerase. Virology 2004 Jun 20; 324 (1): 84-9

27. Wu CJ, Huang HW, Liu CY, et al. Inhibition of SARS-CoV replication by siRNA. Antiviral Res 2005 Jan; 65 (1): 45-8

28. Ni B, Shi X, Li Y, et al. Inhibition of replication and infection of severe acute respiratory syndrome-associated coronavirus with plasmid-mediated interference RNA. Antivir Ther 2005; 10 (4): 527-33 
29. Zhao P, Qin ZL, Ke JS, et al. Small interfering RNA inhibits SARS-CoV nucleocapsid gene expression in cultured cells and mouse muscles. FEBS Lett 2005 Apr 25; 579 (11): 2404-10

30. Shi Y, Yang de H, Xiong J, et al. Inhibition of genes expression of SARS coronavirus by synthetic small interfering RNAs. Cell Res 2005 Mar; 15 (3): 193-200

31. Zheng BJ, Guan Y, Tang Q, et al. Prophylactic and therapeutic effects of small interfering RNA targeting SARS-coronavirus. Antivir Ther 2004 Jun; 9 (3): $365-74$

32. Li BJ, Tang Q, Cheng D, et al. Using siRNA in prophylactic and therapeutic regimens against SARS coronavirus in Rhesus macaque. Nat Med 2005 Sep; 11 (9): 944-51

33. He ML, Zheng BJ, Chen Y, et al. Kinetics and synergistic effects of siRNAs targeting structural and replicase genes of SARS-associated coronavirus. FEBS Lett 2006 May 1; 580 (10): 2414-20

34. Elmen J, Thonberg H, Ljungberg K, et al. Locked nucleic acid (LNA) mediated improvements in siRNA stability and functionality. Nucleic Acids Res 2005; 33 (1): 439-47

35. Meng B, Lui YW, Meng S, et al. Identification of Effective siRNA blocking the expression of SARS viral envelope E and RDRP genes. Mol Biotechnol 2006 Jun; 33 (2): 141-8

36. Ruan YJ, Wei CL, Ee AL, et al. Comparative full-length genome sequence analysis of 14 SARS coronavirus isolates and common mutations associated with putative origins of infection. Lancet 2003 May 24; 361 (9371): 1779-85

37. Khvorova A, Reynolds A, Jayasena SD. Functional siRNAs and miRNAs exhibit strand bias. Cell 2003 Oct 17; 115 (2): 209-16

38. Schwarz DS, Hutvagner G, Du T, et al. Asymmetry in the assembly of the RNAi enzyme complex. Cell 2003 Oct 17; 115 (2): 199-208

39. Aronin N. Target selectivity in mRNA silencing. Gene Ther 2006 Mar; 13 (6): 509-16

40. Poon LL, Leung CS, Chan KH, et al. Recurrent mutations associated with isolation and passage of SARS coronavirus in cells from non-human primates. J Med Virol 2005 Aug; 76 (4): 435-40
41. Wu HL, Huang LR, Huang CC, et al. RNA interference-mediated control of hepatitis B virus and emergence of resistant mutant. Gastroenterology 2005 Mar; 128 (3): 708-16

42. Bridge AJ, Pebernard S, Ducraux A, et al. Induction of an interferon response by RNAi vectors in mammalian cells. Nature Genet 2003; 34 (3): 263-4

43. Grimm D, Streetz KL, Jopling CL, et al. Fatality in mice due to oversaturation of cellular microRNA/short hairpin RNA pathways. Nature 2006; 441 (7092): $537-41$

44. Koehler DR, Hitt MM, Hu J. Challenges and strategies for cystic fibrosis lung gene therapy. Mol Ther 2001 Aug; 4 (2): 84-91

45. Koehler DR, Sajjan U, Chow YH, et al. Protection of Cftr knockout mice from acute lung infection by a helper-dependent adenoviral vector expressing Cftr in airway epithelia. Proc Natl Acad Sci U S A 2003 Dec 23; 100 (26): 15364-9

46. Koehler DR, Frndova H, Leung K, et al. Aerosol delivery of an enhanced helperdependent adenovirus formulation to rabbit lung using an intratracheal catheter. J Gene Med 2005 Nov; 7 (11): 1409-20

47. Koehler DR, Martin B, Corey M, et al. Readministration of helper-dependent adenovirus to mouse lung. Gene Ther 2006 May; 13 (9): 773-80

48. Cao HB, Wang A, Martin B, et al. Down-regulation of IL-8 expression in human airway epithelial cells through helper-dependent adenoviral-mediated RNA interference. Cell Res 2005 Feb; 15 (2): 111-9

49. Schiffelers RM, Storm G. ICS-283: a system for targeted intravenous delivery of siRNA. Expert Opin Drug Deliv 2006 May; 3 (3): 445-54

50. Dykxhoorn DM, Lieberman J. Knocking down disease with siRNAs. Cell 2006 Jul 28; 126 (2): $231-5$

Correspondence: Dr Jim Hu, Lung Biology Research Program, Hospital for Sick Children, 555 University Avenue, Toronto, ON M5G 1X8, Canada.

E-mail: jim.hu@utoronto.ca 